\title{
Influence of interactive stratospheric chemistry on large-scale air mass exchange in a global circulation model
}

\author{
T. Orgis $(a), *$ \\ S. Brand ${ }^{(a)}$ \\ U. Schwarz ${ }^{(b)}$ \\ D. Handorf ${ }^{(a)}$ \\ K. Dethloff ${ }^{(a)}$ \\ J. Kurths ${ }^{(c)}$
}

May 29, 2009

\begin{abstract}
(a) Alfred-Wegener Institute for Polar and Marine Research - Research Unit Potsdam
(b) Center for Dynamics of Complex Systems at the University of Potsdam

(c) Potsdam Institute for Climate Impact Research
\end{abstract}

\begin{abstract}
A new globally uniform Lagrangian transport scheme for large ensembles of passive tracer particles is presented and applied to wind data from a coupled atmosphere-ocean climate model that includes interactive dynamical feedback with stratospheric chemistry. This feedback from the chemistry is found to enhance large-scale meridional air mass exchange in the northern winter stratosphere as well as intrusion of stratospheric air into the troposphere, where both effects are due to a weakened polar vortex.
\end{abstract}

Original publication / citation:

European Physical Journal Special Topics, vol. 174,

doi : 10.1140/epjst/e2009-01105-8

\section{Introduction}

The topic of this study is the analysis of large-scale transport patterns in the atmosphere over seasonal time scales. Lagrangian trajectory ensembles are the means of choice, especially with the computing resources available today. Indeed, modeling transport of passive air parcels is a task that scales very well with distributed/clustered computer architectures (the current de facto standard in high performance computing) as each subset of trajectories in an ensemble can be computed independently of the others.

While e.g. [Sutton 1994] already employed Lagrangian transport on seasonal and global scales, with focus on higher atmospheric layers, the scale of experiments possible today, with some days of CPU time even on small clusters (below 50 AMD Opteron CPUs), is a different one: Instead of Sutton's ensemble size of around 50,000 particles, this study deals with many millions of individual particle trajectories from fully three-dimensional computation, using provided horizontal and vertical wind components.

Lagrangian trajectories are always only an infinitesimal probing of the "real" air dynamics. Still, increasing the number of probes is a priority to improve coverage of possible transport paths - and

${ }^{*}$ Contact via eMail: thomas. orgis@awi.de 
in this case, the actual quantification of air transport and mixing between certain source and target regions.

The subject to be analyzed for its transport properties is the model data resulting from runs of the coupled atmosphere-ocean climate model described in [Brand 2008], which incorporates dynamical feedback coupling with stratospheric chemistry. The question is: What influence on large-scale air mass exchange does the interactive stratospheric chemistry exert?

For assessing that question, the focus is on the change in atmospheric air mass transport from polar regions into lower latitudes and intrusion of stratospheric air into the troposphere. It is investigated how the important transport barriers of the polar vortexes and at the tropopause are altered in the improved general circulation model.

After an introduction to the climate model and the data which was used to compute transport, as well as a brief discussion of relevant differences in the respective two model runs within section 2 , the global transport method itself is presented in section 3. The two main computer experiments investigating changes in large-scale air mass exchange are described in section 4 , along with their results. Finally, section 5 consists of a summary and conclusions.

\section{Data}

\subsection{ECHO-GiSP: Global circulation model and simulations}

Wind data from two long term climate simulations with the coupled AOGCM ${ }^{1}$ ECHO-GiSI ${ }^{2}$ have been used for the transport experiments in this study. ECHO-GiSP, as described by [Brand 2008], is a development based on the coupled AOGCM ECHO-G [Legutke and Voss 1999]. Essentially, the middle atmosphere version [Manzini and McFarlane 1998] of the atmospheric model part (ECHAM, [Roeckner et al. 1996]) was used, and in particular the model was also extended by an interactive chemistry module based on the MECCA chemistry module [Sander et al. 2005]. This enables a detailed discussion of two way feedback loops between stratospheric chemistry, which is mainly a stratospheric ozone chemistry, and atmospheric circulation within a coupled AOGCM.

Technically, there are 39 model levels in the atmosphere, which have been interpolated to 23 pressure levels between $1000 \mathrm{hPa}$ and $0.01 \mathrm{hPa}$ for the transport computations. Horizontally, a spectral T30 resolution has been employed for the atmosphere with $3.75^{\circ}$ longitude resolution and around $3.7^{\circ}$ latitude resolution in grid space. Furthermore, the ocean-sea ice component (HOPE-G, [Wolff et al. 1997]) used 21 vertical levels and a horizontal resolution of T42 with a refinement towards the equator, and for heat and freshwater exchange between the atmosphere- and the ocean component a flux correction was applied.

The ECHO-GiSP interactive chemistry scheme includes mainly gas phase and photolysis reactions, but also heterogeneous reactions on polar stratospheric clouds. On the whole, 39 chemical species and 116 reactions were used for the ECHO-GiSP simulations analyzed in this paper. The species include the main members of the $\mathrm{O}_{x}, \mathrm{NO}_{x}, \mathrm{ClO}_{x}, \mathrm{HO}_{x}$ and $\mathrm{BrO}_{x}$ chemical families, as well as gases like $\mathrm{CO}, \mathrm{CO}_{2}, \mathrm{CH}_{4}, \mathrm{~N}_{2}, \mathrm{H}_{2}, \mathrm{H}_{2} \mathrm{O}$. Focusing on stratospheric chemistry, data from the KASIMA chemistry transport model [Kouker et al. 1999] was used in the troposphere to provide boundary conditions there. With this setup, prescribing the chemistry in the troposphere, the scheme is simplified but still allows interactive chemistry and dynamical feedback in the stratosphere.

Using the described scheme, two long-term simulations of 150 years each were performed as timeslice experiments under fixed present-day conditions. While both of them were fully coupled atmosphere-ocean-sea ice runs including stratospheric chemistry, only their treatment of the trace gas concentrations within the ECHO-G radiation scheme [Morcrette 1991] differed. The so called coupled run, denoted as COUP, took into account the interactive chemistry-dynamics coupling, which means

\footnotetext{
${ }^{1}$ Atmosphere-Ocean General Circulation Model

${ }^{2}$ ECHO-G with Integrated Stratospheric chemistry by AWI Research Unit Potsdam
} 
that the radiation scheme was driven by the simulated stratospheric fields of the chemical constituents. On the other hand, the reference run (REF) used prescribed climatological trace gas fields, so that in this case the dynamical variables did not depend on the simulated chemistry.

\subsection{Dynamical changes}

A central structure for global air mass exchange is the polar vortex, being a meridional barrier and forming a dynamical connection between middle atmosphere and troposphere. For the coupled model run, a weakened polar vortex has been been diagnosed in [Brand 2007]. The December to February (DJF) mean zonal wind difference for the Northern Hemisphere (NH) winter vortexes of both runs, averaged over the last 120 years, is in the order of $10 \mathrm{~m} / \mathrm{s}$. This is about one third of the total wind speed relative to the vortex in the reference simulation. For the Southern Hemisphere (SH) winter, the corresponding absolute wind difference between the modeled vortexes is similar, but because of the generally more stable vortex with higher wind speeds, its relative scale is smaller. Therefore, the most significant effects are to be expected for the $\mathrm{NH}$ winter.

It is a known fact that many models overestimate the strength of the polar vortex (see, e.g., [Stenchikov et al. 2006]). In this respect, the consideration of interactive chemistry feedbacks that reduce the strength of the vortex, is an important step for the further improvement of climate models.

The weakening of the stratospheric polar vortex in the coupled model run is connected with a decrease of the strato-mesospheric residual circulation (Brewer-Dobson circulation) through more stable stratification of the stratosphere. This stabilizing effect in the middle atmosphere can be explained by a vertical adjustment of the interactive ozone distribution compared to the prescribed profile in the reference simulation (see upper part of Figure 1). The shifted vertical ozone distribution, especially in lower latitudes, causes more heating at higher altitudes and less heating at lower altitudes (see lower part of Figure 1), leading to an increased stratospheric temperature gradient (see Figure 2), hence more stable stratification.

With a weaker Brewer-Dobson circulation and stratospheric polar vortex, the atmospheric downwelling at the poles, responsible for relatively warm air above, but cold air inside and below the polar vortex, is reduced. Thus, a weaker meridional temperature gradient between pole and tropics evolves in the troposphere. While first of all, this is associated with a weaker tropospheric zonal mean flow, it also provides better conditions for wave propagation, with the potential to amplify the planetary and synoptic scale wave activity in mid-latitudes. Due to wave forcing by vertical propagating tropospheric planetary waves (see [J.G. Charney, P.G. Drazin 1961]), this again weakens the stratospheric polar vortex.

The following Lagrangian particle transport investigations shall determine how far the weakened vortex influences the strength of air mass transport out of polar latitudes and from the stratosphere to the troposphere. A weaker transport barrier leads to the expectation of increased meridional transport between polar and mid latitudes. This could also enhance the deep stratospheric intrusion through the extratropical lowermost stratosphere as described in [Stohl et al. 2003].

\section{Method}

\subsection{Transport computation}

A global passive tracer transport scheme, working with the wind fields from the ECHO-GiSP model runs ${ }^{3}$, has been designed and implemented in form of the computer program package PEP-Tracer (see [Orgis 2007] for a detailed presentation). This scheme uses explicit Lagrangian computation of individual test particle trajectories, aggregated to ensembles of millions, to prepare statements about the movement of air masses from the starting regions of the ensembles.

\footnotetext{
${ }^{3}$ Generally with gridded longitude/latitude/pressure data in NetCDF format.
} 
Orgis et al.- Infl. of interactive strat. chem. on air mass exchange
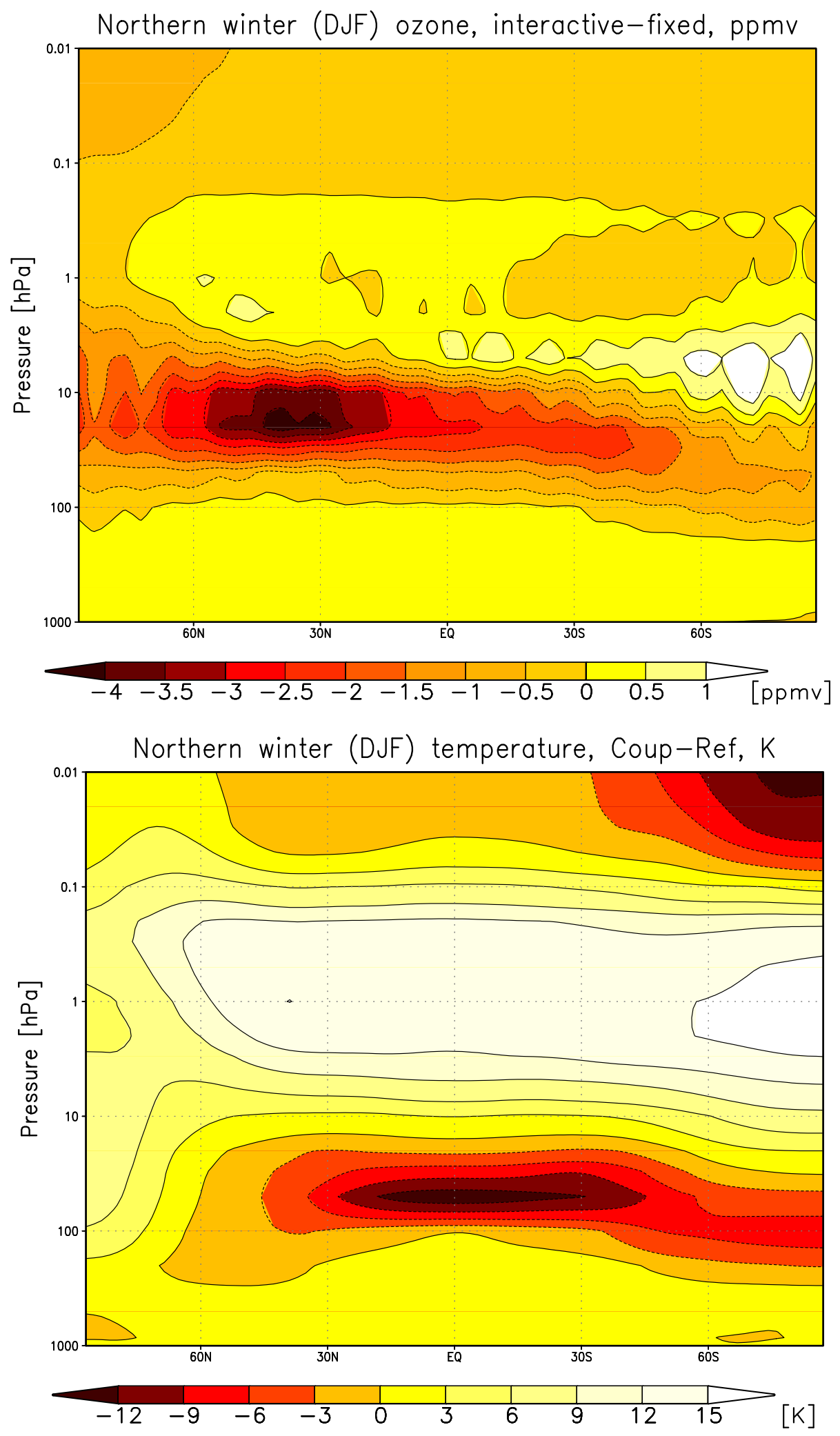

Figure 1: Zonal mean of the difference between the ozone mixing ratio of the coupled simulation and the fixed ozone mixing ratio in the reference simulation on the top, and on the bottom the corresponding temperature difference. The temporal mean is computed over the last 120 simulation years, DJF. The modified ozone distribution causes according changes in the temperature field through absorption of short-wave radiation. 
Orgis et al.- Infl. of interactive strat. chem. on air mass exchange

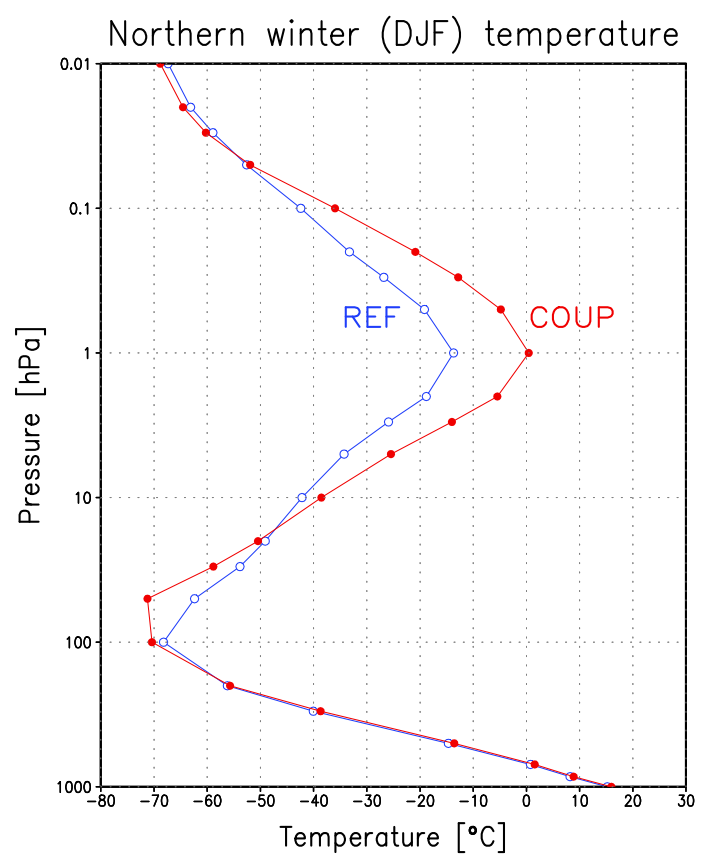

Figure 2: Global mean temperature profiles for coupled and reference run, mean over the last 120 simulation years, DJF. The vertical temperature gradient is enhanced in the coupled run and indicates more stable stratification.

While the wind fields are given in an usual grid with longitude and latitude intervals as horizontal division, the actual advection is computed in a local Cartesian coordinate system for each particle and for each time step. This is a different approach than that used e.g. in the standard FLEXTRA trajectory model [A. Stohl, P. Seibert 1998], where the polar problem is handled by switching to a common stereographic polar projection above a certain latitude. Here, the coordinate transformation is applied at every location. In addition to eliminating the geometric problem near the poles, this allows use of standard time-integration schemes for Cartesian space in a straight-forward way, without modifications.

The local system is a combination of a local orthographic projection ${ }^{4}$ onto the tangent plane with the base point $B=\left(\lambda_{b}, \varphi_{b}, p_{b}\right)$ as the coordinate origin (see Figure 3 ) and an independent barometric transformation of the vertical $p$ coordinate ${ }^{5}$. Any point $P(\lambda, \varphi, p)$ in the neighborhood of $B$ has the coordinates $P^{\prime}(x, y, z)$ in the local Cartesian space (using the radius $R=6370 \mathrm{~km}$ ):

$$
\begin{aligned}
\lambda^{\prime} & =\lambda-\lambda_{p}-\frac{\pi}{2} \\
z(p) & =6715 \mathrm{~m} \cdot \ln \left(\frac{p}{101300 \mathrm{~Pa}}\right) \\
\left(\begin{array}{l}
x \\
y \\
z
\end{array}\right) & =R\left(\begin{array}{c}
\cos \varphi \cdot \cos \lambda^{\prime} \\
\cos \varphi \cdot \sin \lambda^{\prime} \sin \varphi_{p}+\sin \varphi \cos \varphi_{p} \\
z(p)
\end{array}\right) .
\end{aligned}
$$

\footnotetext{
${ }^{4}$ As usual, the earth is treated as perfect sphere in approximation, with the thickness of the atmosphere being neglected for the global geometry.

${ }^{5}$ This is a modification with respect to the original version presented in [Orgis 2007].
} 

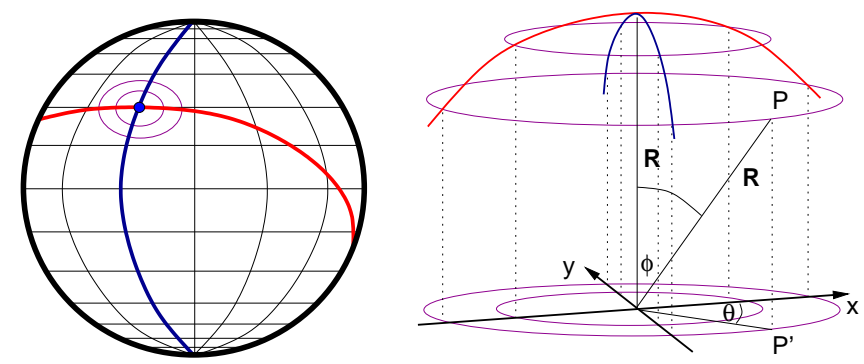

Figure 3: Local orthographic projection near the base point $B$ (center of the concentric circles) from point $P$ in longitude and latitude to the Cartesian point $P^{\prime}$ in $x$ and $y$. The vertical coordinate receives separate treatment.

The horizontal velocity is constructed from the wind components $u$ and $v$ using the transformed base vectors $\vec{e}_{u}$ and $\vec{e}_{v}$ :

$$
\begin{aligned}
& \vec{e}_{u}=\left(\begin{array}{c}
-\sin \lambda^{\prime} \\
\sin \varphi_{p} \cos \lambda^{\prime} \\
0
\end{array}\right), \\
& \vec{e}_{v}=\left(\begin{array}{c}
-\cos \lambda^{\prime} \sin \varphi \\
-\sin \varphi_{p} \sin \lambda^{\prime} \sin \varphi+\cos \varphi_{p} \cos \varphi \\
0
\end{array}\right) .
\end{aligned}
$$

Of course there is some error involved in the chosen local coordinate projection from the curved sphere to a plane. But this error is not systematically increasing towards the poles, where "south" or "north", respectively, can mean many different directions, depending on a displacement of the point of view. The projection error is the same everywhere around the globe, preventing a systematic bias on the transport computation based on the spatial region of that transport. Though, in practice one cannot prevent a systematic error from the varying density of grid points where sampled wind data is given.

The grid cells are not projected to proper rectangles and in the case of the inner polar region, the grid cell is actually a polygon with many vertices (96 with the ECHO-GiSP data), due to the lack of defined wind data at the pole itself. Therefore, one cannot use an interpolation algorithm that depends on a specific regular grid structure. The globally uniform scheme is kept by using the flexible Shepard interpolation ([Shepard 1968], also known as distance weighted sum with the distance function $\left.d\left(\vec{x}, \vec{x}_{i}\right)\right)$ in the local Cartesian horizontal plane. With $n$ surrounding grid points at positions $\left(x_{i}, y_{i}\right)$, the value of a quantity $u$ at $(x, y)$ is then given by

$$
u(\vec{x})=\frac{\sum_{i=1}^{n} \frac{1}{d\left(\vec{x}, \vec{x}_{i}\right)^{k}} u\left(\vec{x}_{i}\right)}{\sum_{i=1}^{n} \frac{1}{d\left(\vec{x}, \vec{x}_{i}\right)^{k}}} .
$$

The exponent for the distance weight was set to $k=2$, as determined optimal by [Shepard 1968]. In $z$ and time direction simple linear interpolation between the respective values resulting from the horizontal interpolation is applied.

In the local space with interpolation values for the wind, the trajectory of the particle currently at the base point $B$ can be computed using several standard algorithms. A four-stage Runge-Kutta as a safe known method has been chosen.

Brief glimpses of the overall advection of air from columns at the poles (extending over the full height of the provided wind data) are shown in Figure 4 . The advection is computed directly at and around the poles naturally like anywhere else with the described method. 

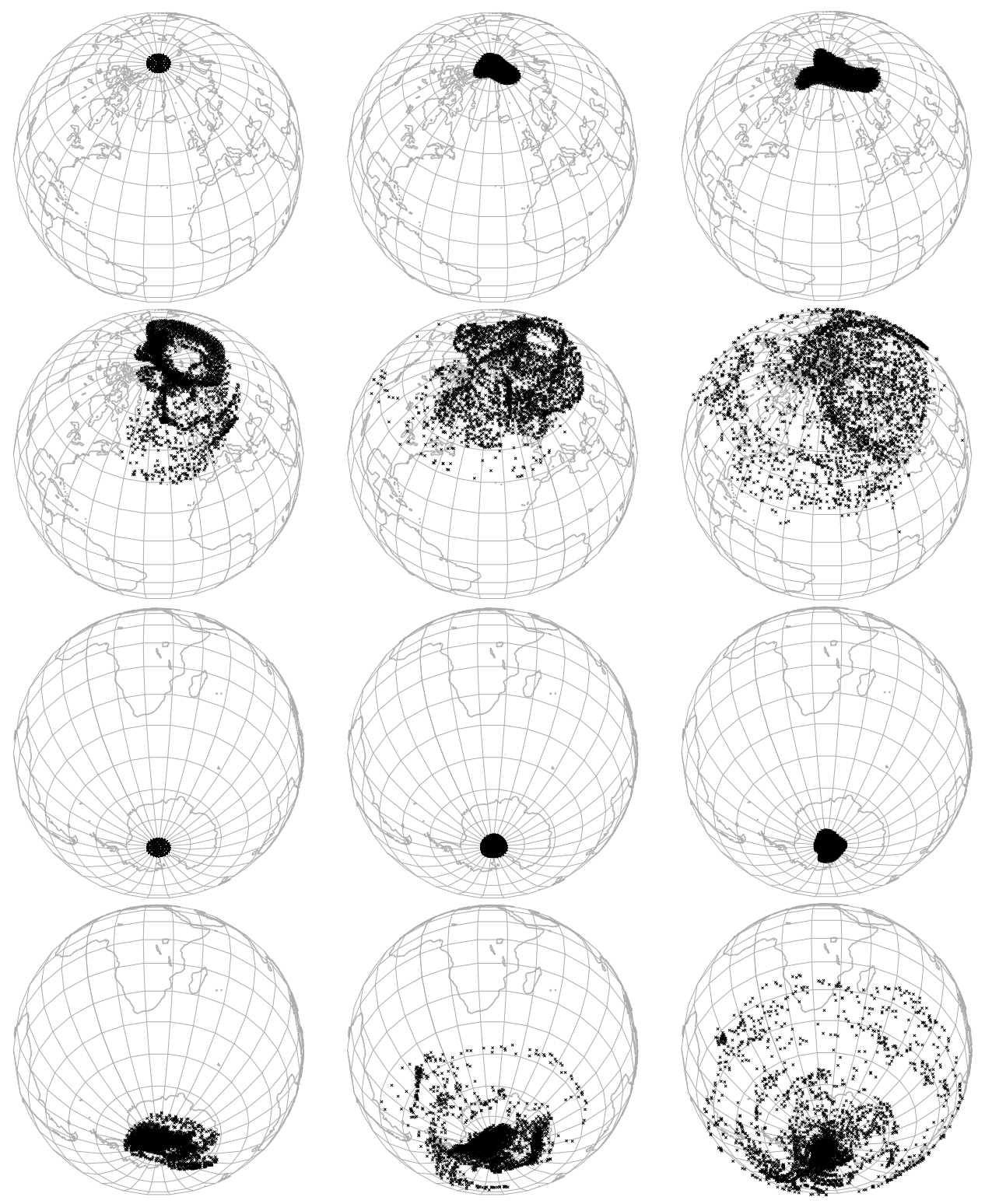

Figure 4: Example column ensembles after 0, 0.2, 0.5, 2, 5 and 10 days for north pole on the top and south pole on the bottom, showing characteristic patterns for the respective pole (during $\mathrm{NH}$ winter). Columns over the polar regions, filled with 3,216 particles $1000 \mathrm{hPa}$ to $0.01 \mathrm{hPa}$, have been advected. The plots show the projection of all particles onto the earth surface, giving a flat view of the horizontal transport dynamics throughout the columns. 
Orgis et al.- Infl. of interactive strat. chem. on air mass exchange
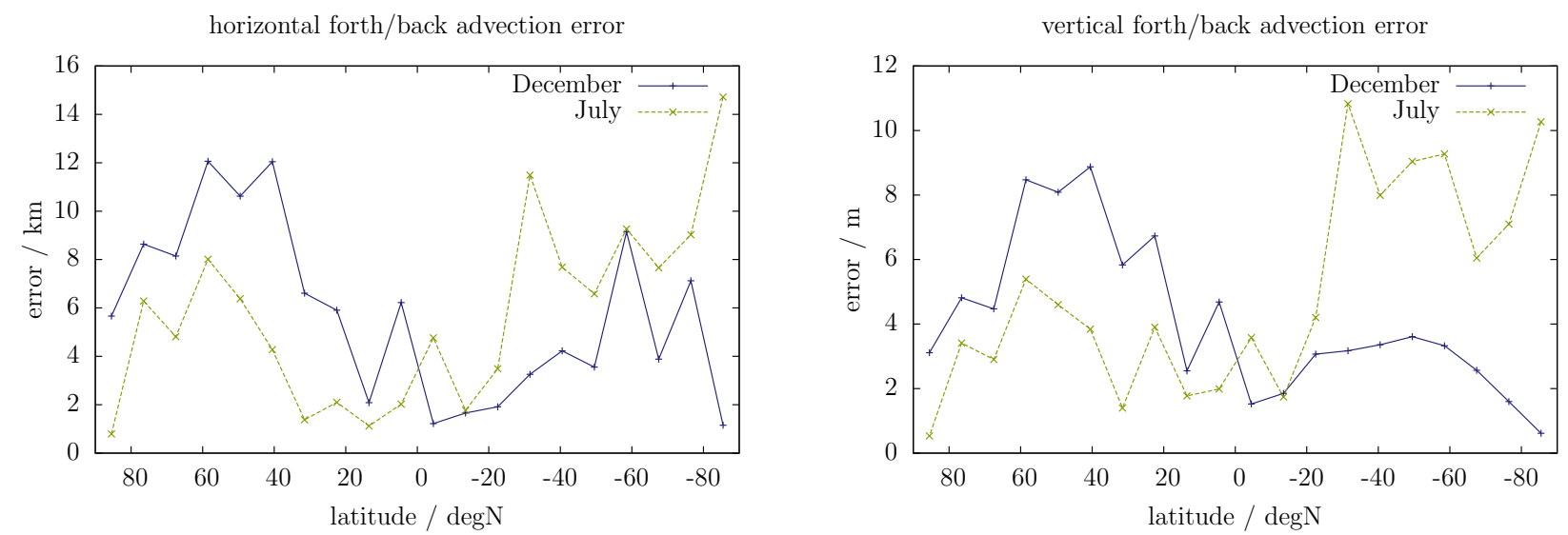

Figure 5: Advection error after five days forth-and-back transport. The plots show the mean error in meters for each latitude, from an ensemble of 4980 particles initially distributed around the globe between $850 \mathrm{hPa}$ and $5 \mathrm{hPa}$. Systematic growth of this error towards the poles cannot be observed. Rather, the seasonal dynamics is indicated when comparing the graph for December and July. Variances are below $100 \mathrm{~km}$ in the horizontal and below $100 \mathrm{~m}$ in the vertical direction, which is still small on the global scale.

Furthermore, a look at the error in forward-backward transport, where particles are advected for a certain time and then back in time to ideally reach their starting point, indeed shows no systematic increase towards the poles. The error rather shows correlation with the dynamic activity in the atmosphere, with larger values towards the winter hemisphere. This is illustrated in Figure 5, where the horizontal error for an ensemble of 4980 particles distributed over the globe in heights from $850 \mathrm{hPa}$ to $5 \mathrm{hPa}$ is shown for December and July of model year sixteen and an one-way transport time of five days. Also, a time step of 0.05 days has been used - corresponding to the largest time step used in the computations later on.

\subsection{Analysis - box counts}

Already under consideration of storage requirements, it is advisable not to store each individual trajectory of large ensembles. For computing mixing ratios and intrusion between regions, counts of particles from certain regions inside certain regions at a certain time form the appropriate data set. The storage size of this array of counts from $n$ source regions in $m$ target regions is virtually independent of the number of particles. The number of count values to be stored is fixed. Only the width of the integral data type must accommodate the maximum possible count value ${ }^{6}$.

A specific horizontal partition scheme eases the comparison of numbers for different regions:

The partition $Z(k, l)$ divides the globe surface into $k$ latitude stripes, each divided into up to $l$ zones. The longitudinal division as well as the latitude thickness is varied for each stripe to produce zones that all cover the same surface area. That way, the numbers (particle counts, resembling air mass) in two zones can immediately be related to each other, respectively the numbers in two sets of zones can be related via the number of zones in each set without further consideration of surface area.

The freedom of choosing the number of zones per stripe makes it possible to keep the thickness of the different stripes close together, yielding - in the limit case for high $k$ and $l$ - an uniform resolution around the globe with zones of same size and similar shape. See Figure 6 for two examples.

A three-dimensional partition $Z m(k, l)$ is constructed by extending the zones to the vertical dimension, by logarithmically dividing the vertical pressure dimension into $m$ layers. Though, one has to

\footnotetext{
${ }^{6}$ So for less than four billion particles, 32 bit unsigned integers are wide enough. 64 bit integers can hold numbers up to ca. $1.8 \cdot 10^{19}$
} 
treat counts of particles from different pressure levels carefully when it comes down to air mass. The exponential variation of air density with height mandates that one can only directly relate region counts from the same level to each other.

\section{Experiments}

Two experiments with passive tracer particle ensembles have been performed:

1. starting ensembles filling polar columns, throughout tropo- and stratosphere

2. starting ensemble filling a layer around the globe at $10 \mathrm{hPa}$.

In both cases the ensembles have been advected for winter and summer seasons, the evolution of the particle distribution indicating the strength of air mass exchange between the starting region and a target region.

Among the 95 years available out of the 150 modeled years, sample time slices have been chosen for the transport investigation, disregarding the first decades to account for the model spin-up.

\subsection{Polar columns}

\subsubsection{Setup}

From the available data, ten DJF (December to February) and ten JJA (June to August) periods in steps of five years from years 25/26 up to 70/71 have been chosen. Starting ensembles were created with an horizontally even distribution of 2930 particles per great circle around the north and south poles, in latitudes up to $66.6^{\circ}$ and 89 pressure levels from $1000 \mathrm{hPa}$ to $1 \mathrm{hPa}$. With the 112923 particles per level, the particle count per column is over $1 \cdot 10^{7}$ for each pole and season. The experiment is set out to compare the coupled and reference runs of ECHO-GiSP, so, altogether, $2 \cdot 2 \cdot 2 \cdot 10 \cdot 1 \cdot 10^{7} \approx 8 \cdot 10^{8}$ individual trajectories have been employed. The column ensembles have been advected using the described PEP-Tracer scheme with a time step of 0.05 days (72 Minutes) over the respective threemonth period.

\subsubsection{Analysis}

The trajectories have been sorted into boxes according to Z30 $(5,3)$, limited to latitudes between $\pm 66.56^{\circ}$ and the respective pole, for the source location and $Z 30(7,13)$ for the target location somewhere on the globe (Figure 6). Based on the counts in these boxes, air mass exchange from the polar region is quantified.

The basic number for each vertical layer is the maximum fraction of the particles having reached beyond $35^{\circ} \mathrm{N}$, or $35^{\circ} \mathrm{S}$, respectively, started from the polar region inside $67^{\circ}$, for the advection period of three months. The final numeric value is the mean over the maximum fraction for the ten individual periods.

In addition, the same counting process has been applied to the number of particles having reached beyond $35^{\circ}$ above $100 \mathrm{hPa}$ or below that vertical separation, respectively (called "COUP above", "REF above" and "COUP below", "REF below" in Figure 7). The three resulting graphs give a basic view on the vertical connection - or lack of - in the polar vortex through $100 \mathrm{hPa}$ together with the horizontal transport out of the polar regions.

Four comparison plots between the control run and the coupled run summarize the result: Air mass exchange from north or south pole, during respective summer or winter.

In Figure 7, the respective summer season for the concerned pole shows little difference. The graphs for the coupled and reference run cling close together, hardly showing any significant difference. Generally, there is mixing of polar particles into lower latitudes approaching $80 \%$ of the particle 

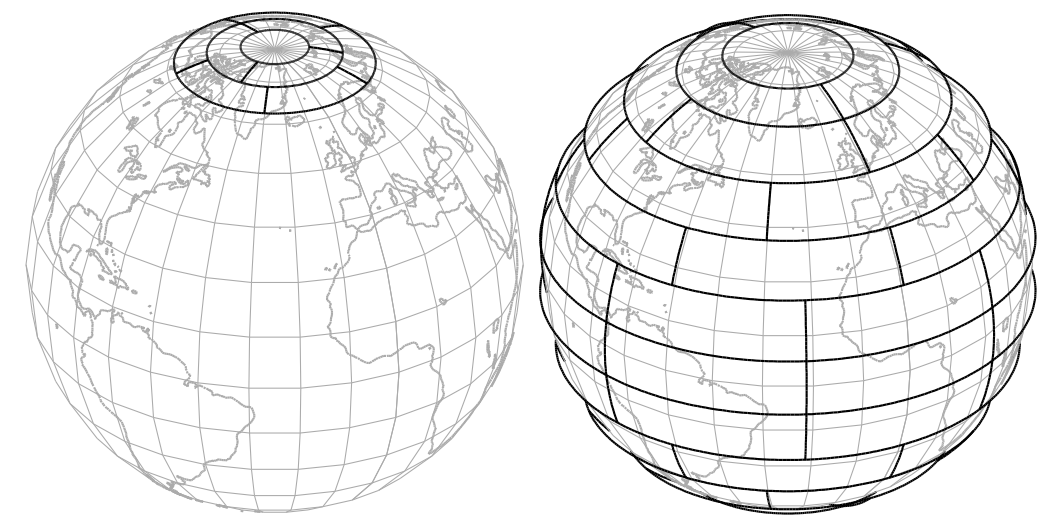

Figure 6: Horizontal zones for polar column experiment. The dark lines depict the borders of the horizontal partition used as basis for box counts of particle locations. The left figure shows the source $Z(5,3)$ partition (restricted to north polar region), the right one the global target $Z(7,13)$. Each zone in one partition covers the same area.

ensemble in the north and $100 \%$ in the south for the upper stratosphere, while the tropospheric mixing is around $50 \%$.

The picture during the respective winter period, shown next to the summer period in Figure 7, is dominated by the strong barrier of the winter polar vortex, generally leading to weaker mixing of stratospheric air into the lower latitudes compared to the summer season. But during the winter period, the effect of the interactive stratospheric chemistry is eminent for the north pole: The magnitude of mixing in the stratosphere is nearly doubled, with about $20 \%$ exchange against the $10 \%$ reference in the upper stratosphere.

To assess the significance of these findings, Figure 7 also includes the mean mixing fractions with the associated statistical error intervals, corresponding to student's t-test $95 \%$ confidence, using the statistics over the ten individual season runs. That test confirms the first main conclusion: The stratospheric mixing in the north polar region, during winter, is significantly enhanced by the stratospheric chemistry coupling of ECHO-GiSP. At the same time, our limited data set does not allow to also draw such a conclusion with statistical confidence for the south polar region.

\subsection{Stratospheric intrusion}

\subsubsection{Setup}

Another basic question is related to the vertical mixing on the global scale, concerning the strength of separation of the different layers in the atmosphere. Especially, the intrusion of stratospheric air into the troposphere, which was also analyzed in detail by [Stohl et al. 2003] using Lagrangian particle dispersion, is of interest.

To investigate this intrusion, a simple experiment employing a global layer of starting positions at $10 \mathrm{hPa}$ has been performed. The horizontal distribution is formed by 10,000 particles per great circle, amounting to an ensemble member count of 31, 828, 494 particles. Each fifteen extended NH winter (November to March) and extended SH winter (May to September) periods, in a 5-year sampling of the available wind data, have been chosen for the advection of the stratospheric ensemble. The ensembles have been advected using the PEP-Tracer scheme with a time step of 0.05 days (72 Minutes) over the respective 150-day period. With the comparison of coupled and reference data for each season, $31,828,494 \cdot 2 \cdot 2 \cdot 15 \approx 1.9 \cdot 10^{9}$ trajectories have been computed. 


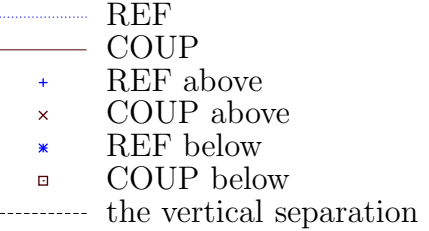

respective summer

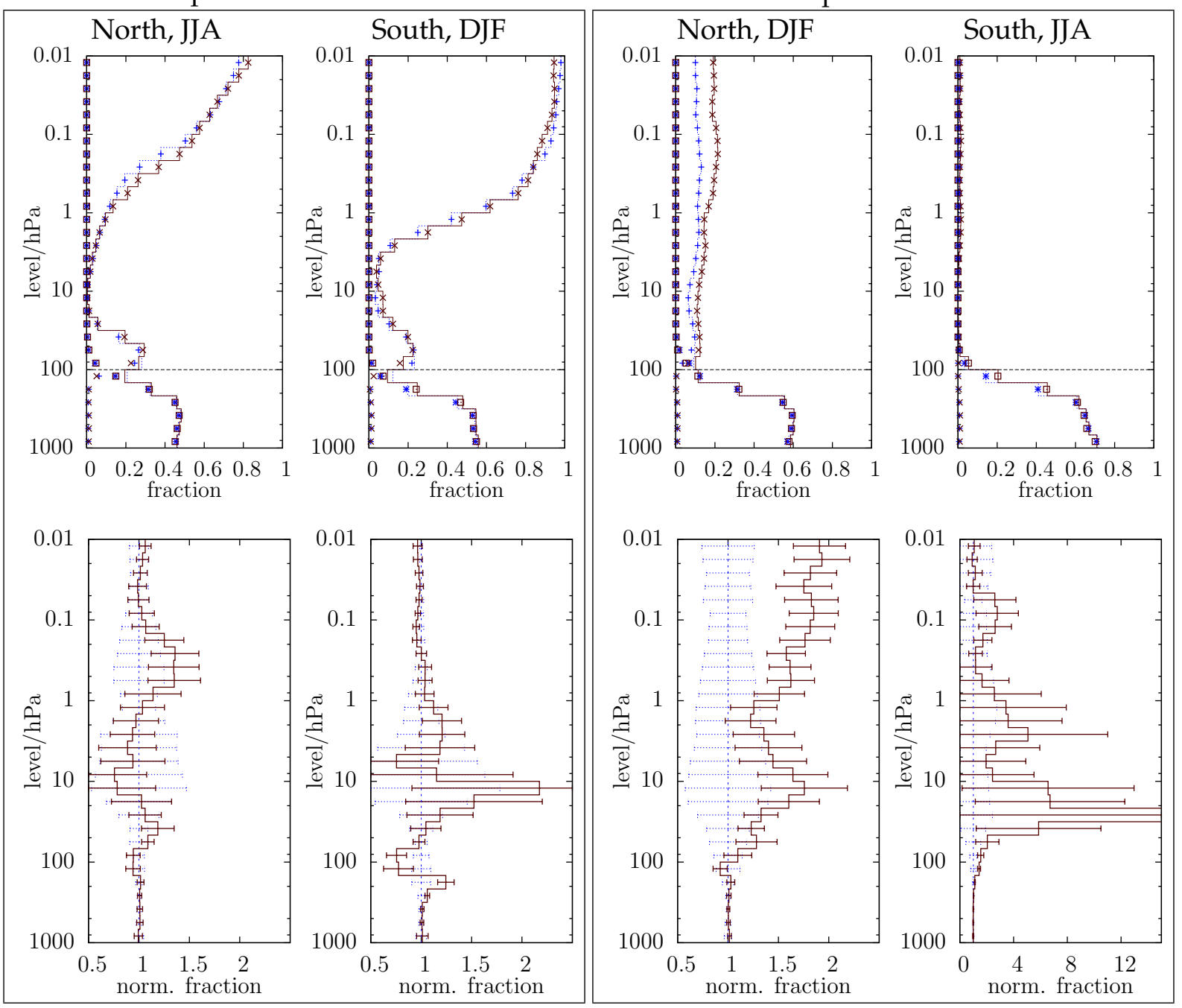

Figure 7: Intrusion to latitudes below $35^{\circ}$ from north and south polar column for each respective summer and winter periods. For every pressure level, the fraction of particles having reached the target region is plotted. The upper part of each panel shows the mean of maximum total fractions per level, the lower part the same mean normalized to the reference, with t-test $95 \%$ confidence intervals. North pole during DJF is the one situation with clearly significant difference between reference and coupled runs. South pole during JJA seems to indicate a similar effect, but the high variance of the small signal does not allow a definite conclusion. 
Orgis et al.- Infl. of interactive strat. chem. on air mass exchange
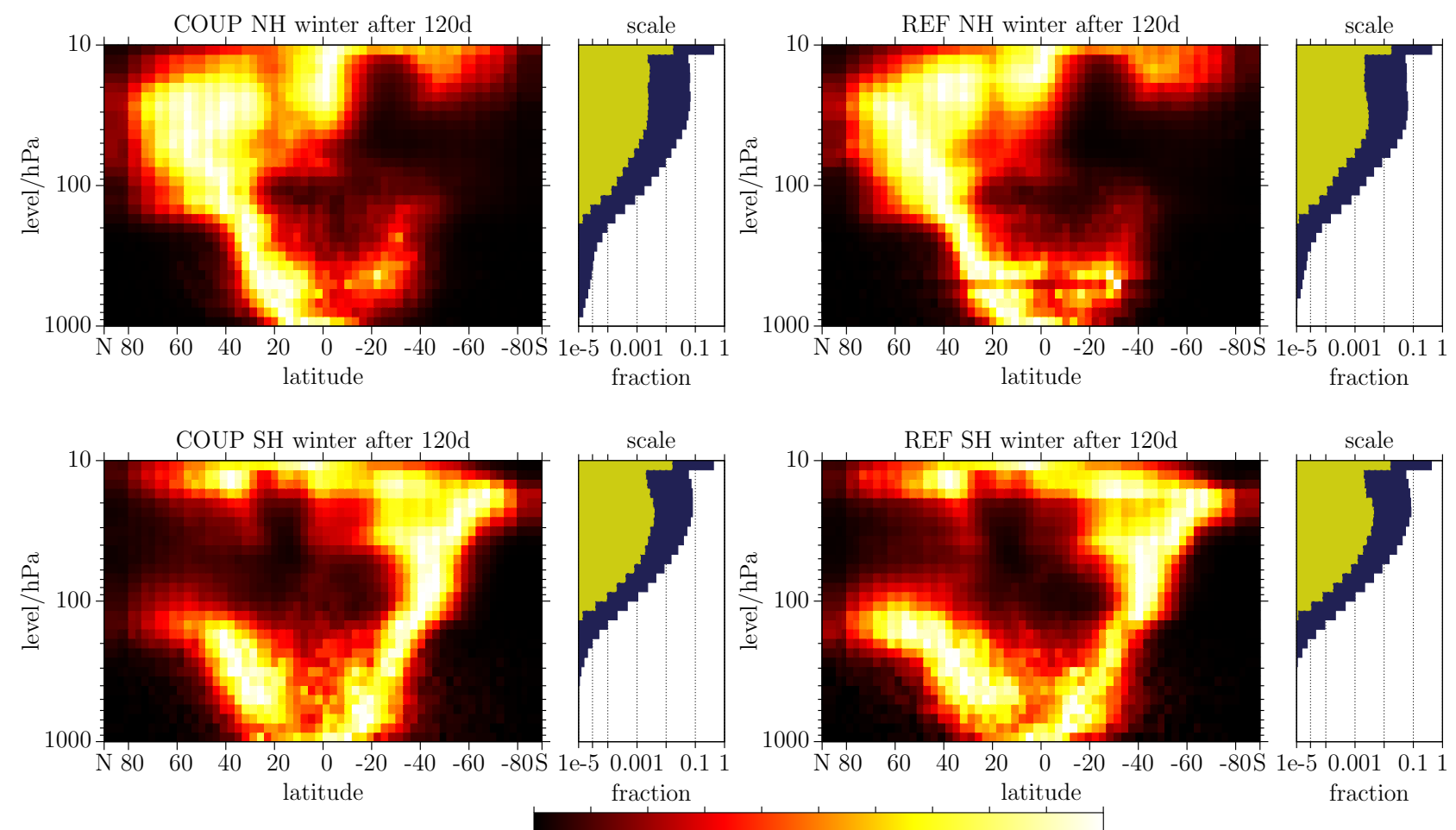

Color map: $0 \begin{array}{lllllllllll}0 & 0.1 & 0.2 & 0.3 & 0.4 & 0.5 & 0.6 & 0.7 & 0.8 & 0.9 & 1\end{array}$

Figure 8: Intrusion after 120 days, comparison for coupled run (left) and reference run (right), $\mathrm{NH}$ winter (top), SH winter (bottom). For each of these four subplots, there is on the left side a zonal view of the structure of distribution of the stratospheric air being advected, normalized to the maximum for each level. Right next to this view is a logarithmic graph of the scale value for each level and the total fraction of ensemble particles in that level. The overall pattern is essentially identical for the coupled and reference runs, but the generally larger amount of intrusion into lower layers during the NH winter is indicated. More interestingly, the increased intrusion for the coupled model data is apparent.

The purpose of this experiment is to quantify the change in intrusion from the stratosphere into the troposphere, but also to show how the tracer transport computation reproduces the global circulation pattern.

\subsubsection{Analysis}

The atmosphere below $10 \mathrm{hPa}$ is the interesting part for this experiment directed at intrusion into the troposphere. Thus, the target partition is a Z30 $(10,60)$ with 30 levels starting below $10 \mathrm{hPa}$. Also, when only global vertical exchange is of interest, the source partition can be just one extended region.

This amount of box counts can be visualized to show the distribution of the stratospheric ensemble over time in a zonal projection. The four plots in Figure 8 summarize the four runs $(\mathrm{NH} / \mathrm{SH}$ winter, coupled/reference) with a snapshot of the distribution after 120 days.

The counts in the partition boxes have been summed along the latitude belts to show the total fraction of particles from the ensemble being contained in a certain latitude-height region. For the picture, all the values in one vertical range are normalized to the maximum fraction in that range, amplifying the small values to make easily visible colors but keeping the latitudinal structure. A bar plot next to the pattern visualization shows the fraction that values in a vertical range have been normalized to as well as the total fraction of the ensemble contained in that vertical range. Together, 
Orgis et al.- Infl. of interactive strat. chem. on air mass exchange
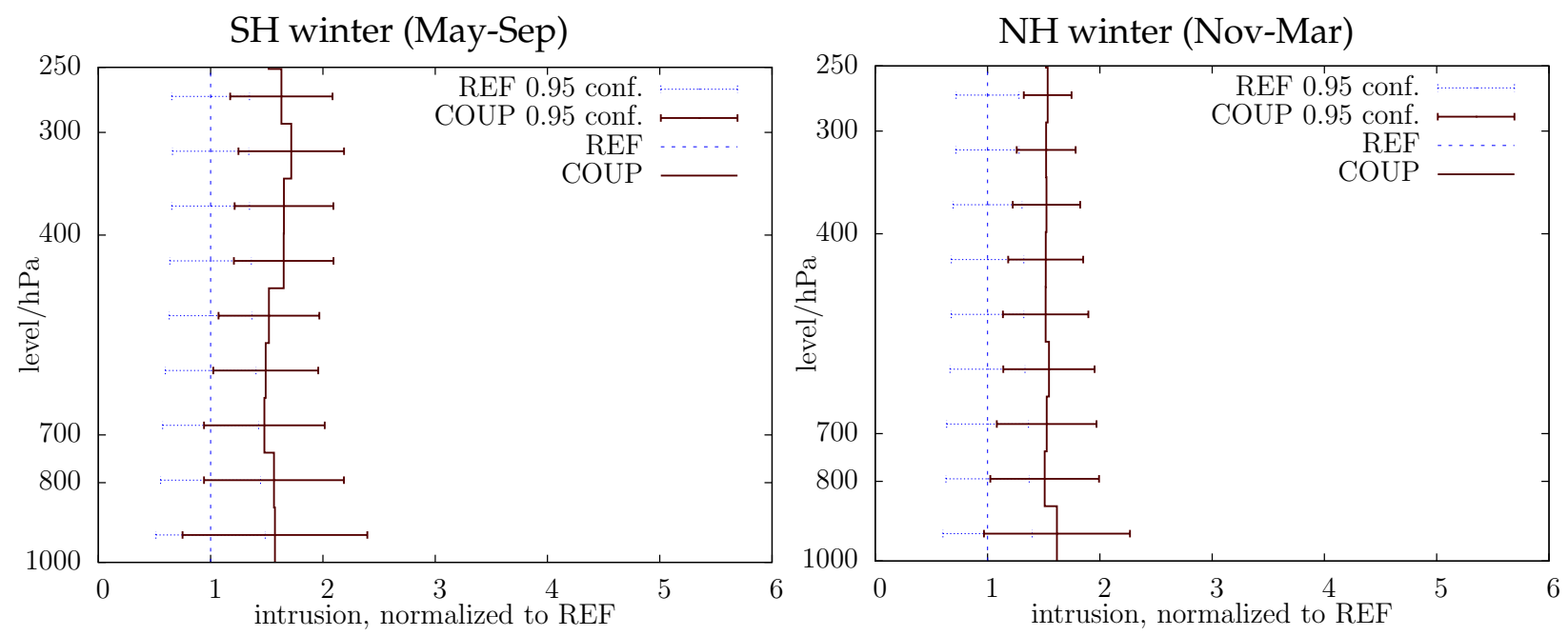

Figure 9: Ensemble mean global amount of stratospheric air in tropospheric layers between $1000 \mathrm{hPa}$ and $250 \mathrm{hPa}$ after 150 days in comparison for coupled and reference run, SH winter and winter. Values are normalized to the reference run, error bars indicate the $t$-test $95 \%$ confidence intervals. The increased intrusion for $\mathrm{NH}$ winter is the most significant signal (at least in the upper troposphere), while the increase for the $\mathrm{SH}$ winter is indicated in a similar scale but closer to the limit of the confidence criterion.

the two sub-plots show the structure and magnitude of the intrusion from the stratosphere into the troposphere.

The NH winter period of the coupled run (top left of Figure 8) features the general trend to downwelling towards the north that is associated with the stratospheric circulation in northern winter. Concerning intrusion, the main transport path is nicely drawn through the tropopause, at around $30^{\circ} \mathrm{N}$. This indicates the mixing through the extratropical lowermost stratosphere as described by [Holton et al. 1995].

Northwards of that, the tropopause shows its strength as a transport barrier: The rectangle from $1000 \mathrm{hPa}$ to $200 \mathrm{hPa}$ and from the north pole to $40^{\circ} \mathrm{N}$ is basically void of any particles. This is similar for the southern hemisphere, but generally transport into regions below $200 \mathrm{hPa}$ is channeled through the north. For the SH winter period of the coupled run (lower left panel of Figure 8), the situation in the stratosphere is reversed, with downwelling towards the south pole as a major pattern.

So far this reproduces a global circulation pattern as expected.

Superficially, the corresponding plots from the reference run (top and bottom right of Figure 8 ) draw the same picture. A difference is in the magnitude of the fractions involved at different heights, observable via the scale graphs. The different strength of intrusion is depicted in detail in Figure 9 , with accompanying confidence intervals to evaluate the significance of the difference.

The number of particles, expressed as fraction of the starting ensemble, that reached levels below $251 \mathrm{hPa}$ at the end of the advection period, is the final number that is analyzed. The results in Table 1 indicate a definite signal from the interactive chemistry: For both seasons, intrusion from the $10 \mathrm{hPa}$ stratosphere is around 50\% stronger for the advection with ECHO-GiSP data with the interactively coupled stratospheric chemistry, compared to the reference without the interactive coupling. The effect is less significant for the $\mathrm{SH}$ winter - being subject to larger relative variance, but the main conclusion is: The intrusion of stratospheric air into the troposphere is enhanced by the stratospheric chemistry coupling of ECHO-GiSP. 
Orgis et al.- Infl. of interactive strat. chem. on air mass exchange

\begin{tabular}{rccc} 
season & coupled \% & reference \%o & coupled/reference \\
\hline NH winter & $0.75 \pm 0.17$ & $0.49 \pm 0.15$ & 1.5 \\
SH winter & $0.26 \pm 0.08$ & $0.16 \pm 0.06$ & 1.6
\end{tabular}

Table 1: Ensemble mean total intrusion into troposphere below $251 \mathrm{hPa}$ after 150 days expressed in terms of fractions of the starting ensemble (ca. $3 \cdot 10^{7}$ particles), with t-test $95 \%$ confidence intervals.

\section{Summary \& Conclusions}

Using the newly developed and freely available Lagrangian transport software package PEP-Tracer [PEP-Tracer], it is possible to compute reliable three-dimensional global passive tracer transport with a consistent interpolation and integration scheme around the globe, with no exception at the poles. Applying this scheme to wind fields provided by the recently developed ECHO-GiSP AOGCM, the influence of the interactively coupled stratospheric chemistry introduced in that model on large-scale transport between the poles and lower latitudes as well as direct intrusion of stratospheric air into the troposphere has been analyzed and quantified.

It has been demonstrated that the interactive chemistry coupling of ECHO-GiSP enhances both the stratospheric air mass exchange from polar latitudes and the intrusion from the extratropical lowermost stratosphere into the troposphere. While the meridional exchange is the primary effect, the intrusion of stratospheric air into the troposphere is mainly a consequence of this. The results of the transport study are consistent with the behavior of the polar vortex, which is a main transport barrier in the stratosphere, in the two analyzed model simulations. The interactive chemistry coupling introduced in ECHO-GiSP results in a weakened polar vortex, the effect of which on global air mass transport is observable using the PEP-Tracer Lagrangian trajectory method. According to the stronger relative change in vortex strength for the $\mathrm{NH}$ winter, the most significant effects are found for that period, focusing on the northern hemisphere.

\section{Acknowledgments}

This work has been funded by the German Helmholtz Association through the Pole-Equator-Pole virtual institute $[\mathrm{PEP}]$. We thank the anonymous reviewers for their helpful comments.

\section{References}

[A. Stohl, P. Seibert 1998]

[Brand 2007]

[Brand 2008]

[Holton et al. 1995]
A. Stohl, P. Seibert, Q.J. Roy. Met. Soc. 124 (1998), pp. 1465-1484, DOI: 10.1002/qj.49712454907

S. Brand, K. Dethloff, D. Handorf, Open Atmos. Sci.J. 1 (2007), pp. 6-19

S. Brand, K. Dethloff, D. Handorf, Geophys. Res. Lett. 35 (2008), pp. L05809, DOI: 10.1029/2007GL032312

J.R. Holton, P.H. Haynes, M.E. McIntyre, A.R. Douglass, R.B. Rood and L. Pfister, Reviews of Geophysics 33 (1995), pp. 403-439

[J.G. Charney, P.G. Drazin 1961] J.G. Charney, P.G. Drazin, J. Geophys. Res. 66 (1961), pp. 83-109, DOI: 10.1029/JZ066i001p00083 
[Kouker et al. 1999]

[Legutke and Voss 1999]

[Manzini and McFarlane 1998]

[Morcrette 1991]

[Orgis 2007]

[PEP]

[PEP-Tracer]

[Roeckner et al. 1996]

[Sander et al. 2005]

[Shepard 1968]

[Stenchikov et al. 2006]

[Stohl et al. 2003]

[Sutton 1994]

[Wolff et al. 1997]
W. Kouker, I. Langbein, Th. Reddmann, R. Ruhnke, Wiss. Berichte, FZKA 6278, FZ Karlsruhe, Germany (1999)

S. Legutke and R. Voss, Technical Report No. 18, ISSN 0940-9327, DKRZ Hamburg, Germany (1999)

E. Manzini, N. A. McFarlan, J. Geophys. Res. 103 (1998), pp. 3152331540

J.J. Morcrette, J. Geophys. Res. 96 (1991), pp. 9121-9132

T. Orgis, Lagrangesche Traceradvektion in einem globalen gekoppelten Klimamodell, http://thomas.orgis.org/diplom/ (2007) (Diploma Thesis, University of Potsdam, Germany)

PEP virtual institute, Pole - Equator - Pole (PEP) - Variability of atmospheric trace constituents along a North-South Transect, http: //www . awi-potsdam. de/www-pot/atmo/pep/(2005-2007)

T. Orgis, PEP-Tracer software package, http://thomas.orgis.org/ pep-tracer/ (2009)

E. Roeckner, K. Arpe, L. Bengtsson, M. Christoph, M. Claussen, L. Dümenil, M. Esch, M.A. Giorgetta, U. Schlese, U. Schulzweida, Report No. 218, MPI for Met. Hamburg, Germany (1996)

R. Sander, A. Kerkweg, P. Jöckel, J. Lelieveld, Atmos. Chem. Phys. 5 (2005), pp. 445-450

D. Shepard, A two-dimensional interpolation function for irregularlyspaced data, Proceedings of the 1968 ACM National Conference (1968), pp. 517-524

G. Stenchikov, K. Hamilton, R.J. Stouffer, A. Robock, V. Ramaswamy, B. Santer, and H.-F. Graf, J. Geophys. Res. 111 (2006), pp. D07107, DOI: 10.1029/2005JD006286

A. Stohl, H. Wernli, M. Bourqui, C. Forster, P. James, M.A. Liniger, P. Seibert, and M. Sprenger, Bull. Am. Met. Soc. 84 (2003), pp. 1565-1573, DOI: 10.1175/BAMS-84-11-1565

R. Sutton, Q.J. R. Meteorol. Soc. 120 (1994), pp. 1299-1321

J.O. Wolff, E. Maier-Reimer, S. Legutke, Technical Report No. 13, ISSN 0940-9327, DKRZ Hamburg, Germany (1997) 